\title{
Análisis de la Fertilidad Potencial del Canino (Canis Lupus Familiaris) Posterior a la Inyección Intratesticular de Cloruro de Calcio al $20 \%$.
}

\author{
Pamela Morales M., M.V., MS.C.; Francisca Contardo O., M.V.; Federico Cifuentes R., M.V, \\ MS.C.; Claudia Rojas Z., M.V., MS.C.
}

Facultad de Recursos Naturales y Ciencias Veterinarias, Escuela de Medicina Veterinaria, Universidad Santo Tomás, sede Talca, Avda. Carlos Schorr 255, Talca.

\begin{abstract}
Resumen
Una de las principales técnicas utilizadas para el control de la población canina es la castración. La técnica quirúrgica sin lugar a dudas es la más utilizada y conocida, sin embargo la opción de la castración química ha ido tomando fuerza como una alternativa a la castración quirúrgica, debido a que se considera un proceso no invasivo, económico y de fácil realización, sobre todo en casos de castración masiva de animales como control de la población callejera. En este estudio se utilizó un total de 20 caninos, los cuales se dividieron en: grupos experimentales $(1,2,3)$ y grupo control. A los tres grupos experimentales se les aplicó una inyección por vía intratesticular de cloruro de calcio al $20 \%$ en ambos testículos, mientras que, al grupo control se le inyectó por la misma vía, solución fisiológica al $0,9 \%$. Previo a esta inoculación todos los pacientes fueron sometidos a un examen físico general, examen andrológico, análisis de fertilidad mediante espermiograma y análisis plasmático de testosterona mediante radio inmuno ensayo (RIA). Al grupo experimental 1 y al grupo control, luego de 7 días post inoculación, se les realizó un nuevo análisis de fertilidad mediante espermiograma y análisis plasmático de testosterona. En el caso del grupo experimental 2 el procedimiento anterior se realizó después de 14 días de la inoculación y el grupo experimental 3, después de 21 días. En relación con el análisis espermático, el grupo experimental 1 no presentó diferencias significativas en ningún parámetro analizado. El grupo experimental 2 presentó diferencias estadísticamente significativas en volumen, concentración espermática y motilidad progresiva. El grupo experimental 3 presentó diferencias estadísticamente significativas en el volumen entre el tiempo inicial y a los 21 días posterior a la inoculación, los parámetros restantes del espermiograma no fueron analizados puesto que no se obtuvo líquido seminal en los pacientes de este grupo, indicando que a los 21 días existiría daño en el tejido testicular evidenciado por la ausencia de espermatogénesis. Los resultados del análisis plasmático de testosterona indicaron que solo en el grupo experimental 3 la concentración de ésta hormona presentó una disminución estadísticamente significativa, por lo cual a partir de los 21 días post-inoculación la concentración de esta hormona comenzaría a descender. Debido a los resultados obtenidos podemos concluir que la utilización de cloruro de calcio al $20 \%$ vía intratesticular es una técnica eficaz en lograr la castración, lo que se manifiesta con mayor seguridad a partir de los 21 días post inyección, fácil de realizar y sin efectos adversos, lo que la hace una opción válida, sobre todo en planes de esterilización masiva.
\end{abstract}

Palabras clave: Castración; Fertilidad; Perros.

\section{Introducción}

Los testículos corresponden a la gónada masculina, su parénquima posee gran cantidad de túbulos seminíferos con un epitelio estratificado especializado que contiene células sustentaculares (Sertoli) y células espermatogénicas, estas últimas se diferencian para generar los espermatozoides. En los espacios intertubulares se encuentran las células intersticiales (Leydig), las cuales sintetizan testosterona (Dellmann, 1994; Gartner y Hiatt, 2002; Geneser, 2002; Cunningham, 2003). 
Una evaluación de aptitud reproductiva, incluye examen de los genitales, medición testicular, palpación testicular y prostática y evaluación seminal (Gradil et al., 2006). La evaluación del semen se lleva a cabo después del examen clínico del animal y las características más comúnmente evaluadas son: color, olor, $\mathrm{pH}$, volumen, concentración espermática, motilidad progresiva, motilidad individual $\mathrm{y}$ morfología de los espermatozoides (Corti, 2003).

Para la comunidad, las autoridades y para los médicos veterinarios, es importante el control de la población canina. La sobrepoblación de caninos callejeros, acusa una falta de sistemas eficientes para el control y manejo de la natalidad (Navarrete, 1997; Santos, 2007; Seremi de Salud Región del Maule, 2008). Una de las principales técnicas utilizadas en la actualidad para controlar la natalidad es la castración mediante técnica quirúrgica, la cual provoca un cambio hormonal y conductual en el canino, sin embargo se requiere de infraestructura especial para realizarla, profesionales con experiencia y cuidados post-quirúrgicos del paciente, que hacen que la técnica tenga un costo relativamente alto. Por tales motivos, la opción de castración química ha ido tomando fuerza como una alternativa, debido a que se considera un proceso no invasivo, económico y de fácil realización, sobre todo en casos de castración masiva de animales, las cuales generalmente se realizan a nivel municipal (Jana y Samanta, 2007). La hipótesis del presente estudio es que la aplicación intratesticular bilateral de cloruro de calcio al $20 \%$ en caninos produce infertilidad potencial.

\section{Material y método.}

Este estudio fue realizado en el Hospital Clínico Veterinario de la Universidad Santo Tomás (UST), sede Talca. La unidad biológica utilizada fue el perro doméstico (Canis lupus familiaris). Se utilizaron 20 caninos, correspondientes a mascotas con dueño establecido y pertenecientes al Refugio San Francisco de Asís de Talca. Los dueños o cuidadores de los caninos firmaron un consentimiento informado.

A los caninos se les realizó un examen físico 28 y un examen andrológico general. Posteriormente se les realizó un análisis espermático y se les tomó muestras de sangre para la determinación de los valores plasmáticos de testosterona.
Los criterios de inclusión fueron: caninos machos de 2-5 años. Entre 10-15 kilos. Sin enfermedades testiculares evidentes mediante el examen físico y andrológico general. Fértiles al análisis espermático.

Del total de caninos se constituyeron cuatro grupos de estudio:

- Grupo experimental 1: conformado por 5 caninos que se les aplicó una inyección bilateral intratesticular de $20 \mathrm{mg} / \mathrm{kg}$ de peso de cloruro de calcio al $20 \%$, a los 7 días se les realizó examen espermático y análisis plasmático de testosterona.

- Grupo experimental 2: conformado por 5 caninos que se les aplicó el mismo producto, a los 14 días se les realizó examen espermático y análisis plasmático de testosterona.

- Grupo experimental 3: conformado por 5 caninos que se les aplicó el mismo producto, a los 21 días se les realizó examen espermático y análisis plasmático de testosterona.

- Grupo Control: conformado por 5 caninos a los cuales se les aplicó una inyección bilateral intratesticular de solución fisiológica de $\mathrm{NaCl}$ al $0,9 \%$ en un volumen equivalente al utilizado en el cloruro de calcio de acuerdo a su peso corporal, a los 7 días se les realizó examen espermático y análisis plasmático de testosterona.

Se realizó un análisis de fertilidad mediante espermiograma para lo cual se obtuvieron muestras de semen mediante extracción manual antes y después de la inoculación del producto según los grupos. Solo se utilizó la fracción espermática del semen, la cual fue evaluada en cuanto a volumen, $\mathrm{pH}$, motilidad progresiva y concentración espermática, mediante la utilización de microscopio óptico (Modelo N-200 M), tubos de microcentrífuga, cámara de Neubauer y tinción de Giemsa. Además se les extrajo 1 a $2 \mathrm{ml}$ de sangre, antes y después de la aplicación del producto, para la determinación de los valores plasmáticos de testosterona mediante técnica de Radio inmunoensayo (RIA), método estandarizado para la especie canina. Las muestras de sangre se depositaron tubos sin aditivos y mediante centrifugación se separó el suero el cual fue congelado a $-18^{\circ} \mathrm{C}$ hasta su análisis, el cual fue realizado en el Laboratorio de la Pontificia Universidad Católica de Chile.

El protocolo para el procedimiento en todos los animales fue el siguiente: se administró Xilacina al 
$2 \%$ a dosis de $0,5 \mathrm{mg} / \mathrm{Kg}$ junto con atropina a dosis de $0,04 \mathrm{mg} / \mathrm{kg}$ por vía intramuscular. Se administró Tramadol a dosis de $2 \mathrm{mg} / \mathrm{Kg}$ por vía intravenosa. Se depiló la zona testicular y posteriormente se desinfectó con povidona yodada. Se inyectó $20 \mathrm{mg} / \mathrm{kg}$ de cloruro de calcio al $20 \%$ o suero físiológico por vía intratesticular, la inyección fue aplicada desde el polo craneal del testículo hasta la extremidad caudal de él, liberando la solución de forma uniforme.

Los resultados fueron expresados en términos de porcentajes, promedios y desviación estándar. Se utilizó ANDEVA y la prueba estadística de Tukey. Si los datos no respondían a los supuestos de normalidad, se aplicó la prueba no paramétrica de Kruskal-Wallis. Se consideró una significancia de $\mathrm{p} \leq 0,05$.

\section{Resultados.}

Los resultados del espermiograma indican que el promedio de volumen espermático en el grupo control fue de $3 \mathrm{ml}$. tanto antes como a los 7 días posterior a la inoculación de solución fisiológica. En el grupo experimental 1 el volumen presentó un promedio de $2,5 \mathrm{ml}$. antes y a los 7 días de la inoculación de cloruro de calcio, no existiendo diferencia significativa entre estos tiempos. En relación al grupo experimental 2 el volumen inicial presentó un promedio de $2,5 \mathrm{ml}$ y a los 14 días posteriores a la inoculación de 2,0 $\mathrm{ml}$, indicando diferencia significativa. Finalmente, los resultados del análisis de volumen espermático en el grupo experimental 3, mostraron una promedio inicial de $2,5 \mathrm{ml}$ y a los 21 días posteriores a la inoculación del producto de $0 \mathrm{ml}$., existiendo diferencia estadísticamente significativa entre los tiempos (Tabla 1).

Los resultados del análisis de $\mathrm{pH}$ espermático indican que en el tiempo inicial y en los tiempos 7 y 14 posterior a la inoculación del producto el promedio de este parámetro fue de 6,0 , no indicando diferencia significativa entre los tiempos. Sin embargo el grupo experimental 3 a los 21 días de la inoculación no se obtuvo líquido seminal por lo cual no se realizó dicha medición (Tabla 1).

Los resultados del análisis de la concentración espermática por $\mathrm{ml}$ en el grupo control, mostraron un promedio en el tiempo inicial de $1,7 \times 10^{8}$ espermatozoides y a los 7 días posteriores a la inoculación de solución fisiológica de 7,4 x $10^{7}$ espermatozoides no observando diferencia significativa.

\begin{tabular}{|c|c|c|c|c|}
\hline Parámetros & $\begin{array}{c}\text { Grupo } \\
\text { Control } \\
\text { (7días) }\end{array}$ & $\begin{array}{l}\text { Grupo } \\
\text { Exp. } 1 \\
\text { (7días) }\end{array}$ & $\begin{array}{c}\text { Grupo } \\
\text { Exp. } 2 \\
\text { (14días) }\end{array}$ & $\begin{array}{c}\text { Grupo } \\
\text { Exp. } 3 \\
\text { (21días) }\end{array}$ \\
\hline $\begin{array}{l}\text { Volumen } \\
\text { seminal }\end{array}$ & S.D & S.D & $\downarrow$ & $\downarrow$ \\
\hline pH seminal & S.D & S.D & S.D & N.O \\
\hline $\begin{array}{l}\text { Concentración } \\
\text { espermática }\end{array}$ & S.D & S.D & $\downarrow$ & N.O \\
\hline $\begin{array}{l}\text { Motilidad } \\
\text { Masal }\end{array}$ & S.D & S.D & $\downarrow$ & N.O \\
\hline $\begin{array}{l}\text { Circunferencia } \\
\text { escrotal }\end{array}$ & S.D & $\downarrow$ & $\downarrow$ & $\downarrow$ \\
\hline
\end{tabular}

Los resultados del grupo experimental 1 , indicaron un promedio de concentración espermática en el tiempo inicial de $5 \times 10^{7}$ espermatozoides y a los 7 días posteriores a la inoculación de cloruro de calcio de 2,5 x $10^{7}$ espermatozoides, sin diferencia entre los tiempos. Respecto al grupo experimental 2 los resultados de la concentración espermática en este grupo mostraron un promedio en el tiempo inicial de $4,5 \times 10^{7}$ espermatozoides y a los 14 días posteriores a la inoculación de 5,5 x $10^{6}$ espermatozoides, indicando diferencia estadísticamente significativa. Por otra parte, los resultados del análisis de la concentración espermática en el grupo experimental 3 , mostraron un promedio en el tiempo inicial de $2,2 \mathrm{x}$ $10^{8}$ espermatozoides y a los 21 días posterior a la inoculación no se pudo realizar esta medición ya que no se obtuvo líquido seminal (Tabla 1).

Los resultados del análisis de la motilidad progresiva en el grupo control indican un promedio en el tiempo inicial de $78 \pm 10,95 \%$ y a los 7 días posteriores a la inoculación de solución fisiológica de $70 \pm 14,14 \%$, no existiendo diferencia entre los tiempos.

En el grupo experimental 1 se obtuvo un promedio en el tiempo inicial de $82 \pm 10,95 \%$ y a los 7 días posteriores a la inoculación de cloruro de calcio de 78 $\pm 10,95 \%$, sin diferencia significativa.

En el grupo experimental 2 el promedio inicial fue de $66 \pm 16,73 \%$ y a los 14 días posteriores a la inoculación de $34 \pm 19,49 \%$, indicando diferencia entre los tiempos. 
Finalmente en el grupo experimental 3 el promedio inicial fue de $86 \pm 8,94 \%$ y a los 21 días posteriores a la inoculación del producto no se pudo realizar esta medición ya que no se obtuvo líquido seminal (Tabla $1)$.

Al realizar una comparación entre los grupos, podemos indicar que el grupo experimental 2 presentó una disminución significativa en la concentración espermática a los 14 días en comparación con los otros grupos en estudio.

En relación a la circunferencia escrotal, el grupo control presentó un promedio inicial de 15,54 $\pm 1,50$ $\mathrm{cm}$ y a los 7 días posteriores de la inoculación de solución fisiológica de $16 \pm 1,90 \mathrm{~cm}$, sin diferencia significativa.

En el grupo experimental 1 el promedio inicial fue de $14,1 \pm 1,81 \mathrm{~cm}$ y a los 7 días de $13,1 \pm 1,42 \mathrm{~cm}$, sin diferencia entre los tiempos.

En el grupo experimental 2 el promedio inicial fue de $12,5 \pm 1,15 \mathrm{~cm}$ y a los 14 días de $11,6 \pm 0,69 \mathrm{~cm}$, indicando diferencia estadísticamente significativa.

Por último en el grupo experimental 3, el promedio inicial fue de $12,2 \pm 0,92 \mathrm{~cm}$ y a los 21 días posteriores de la inoculación fue de 10,3 $\pm 1,2 \mathrm{~cm}$, presentando diferencia significativa (Tabla 1).

$\mathrm{Al}$ realizar la comparación entre los grupos, podemos indicar que el grupo control presentó diferencia significativa con una mayor circunferencia escrotal al compararlo con los grupos experimentales.

En relación a la concentración plasmática de testosterona, el grupo control presentó un promedio inicial de $2,99 \pm 1,26 \mathrm{nmol} / \mathrm{L}$ y a los 7 días posteriores a la inoculación de solución físiológica de $6,71 \pm 4,47$ $\mathrm{nmol} / \mathrm{L}$., sin diferencia estadísticamente significativa entre los tiempos.

En el grupo experimental 1 el promedio inicial de testosterona fue de $6,76 \pm 5,79 \mathrm{nmol} / \mathrm{L}$ y a los 7 días de 2,69 $\pm 0,93 \mathrm{nmol} / \mathrm{L}$, sin diferencia significativa.

En el grupo experimental 2 el promedio inicial de la hormona fue de $3,57 \pm 1,65 \mathrm{nmol} / \mathrm{L}$ y a los 14 días de $3,352 \pm 2,39 \mathrm{nmol} / \mathrm{L}$., sin diferencia significativa.

En el grupo experimental 3 la concentración inicial de testosterona fue de $8,35 \pm 3,94 \mathrm{nmol} / \mathrm{L}$ y a los 21 días de $2,764 \pm 4,09 \mathrm{nmol} / \mathrm{L}$. En este grupo experimental se observó diferencia altamente significativa entre los tiempos (Gráfico 1).

Al realizar la comparación de los resultados de la concentración plasmática de testosterona entre los grupos, se observa que no existe diferencia estadísticamente significativa entre ellos (Gráfico 2).

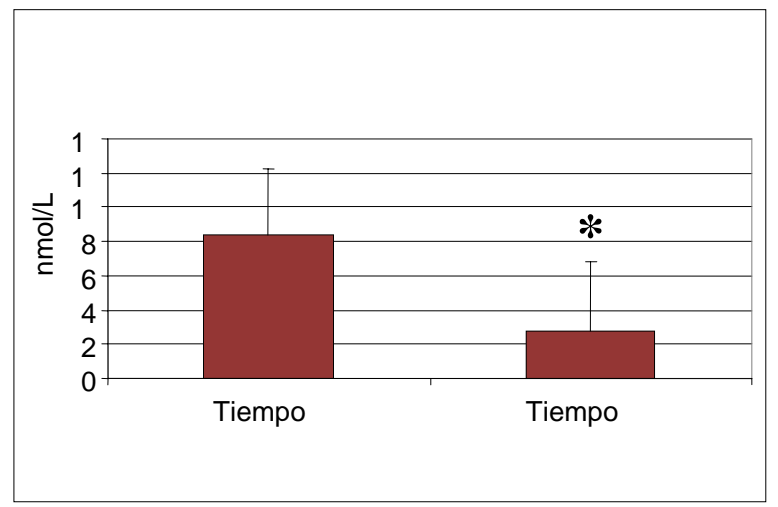

Gráfico $\mathrm{N}^{\circ} 1$. Concentración plasmática de testosterona en el grupo experimental 3. (*) Indica diferencia estadísticamente significativa. $p=0,003$.

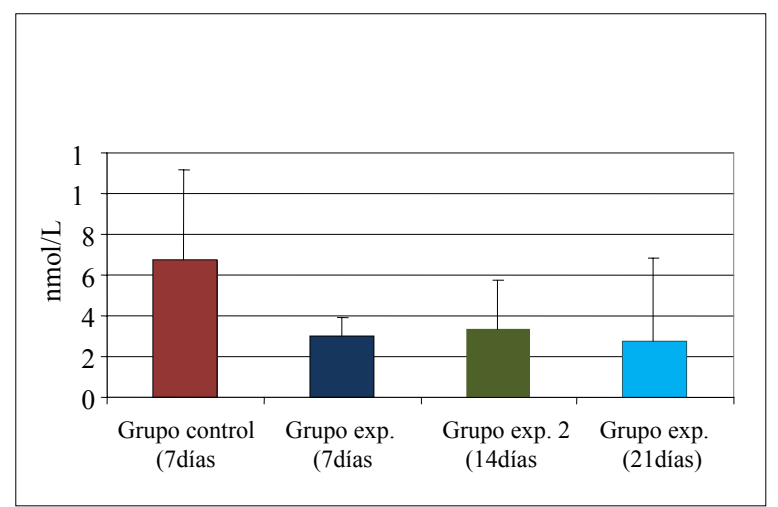

Gráfico $\mathbf{N}^{\circ} 2$. Concentración plasmática de testosterona entre los grupos de estudio.

\section{Discusión.}

Los animales incluidos en este estudio no evidenciaron efectos adversos a la administración del producto, tales como, prurito, dolor y/o inflamación en la región escrotal, como tampoco se observaron signos o síntomas de carácter general como fiebre o depresión, esto difiere de lo descrito por Mora et al., (1991), los cuales utilizaron alfa hidroxipropionico por vía intratesticular en terneros produciendo severa inflamación y adherencias testiculares. 
En el grupo control los parámetros del espermiograma no presentaron diferencias entre los tiempos, lo que indica que la inoculación de solución fisiológica al $0,9 \%$, no altera la calidad del semen y por ende la fertilidad de los animales de este grupo se mantuvo inalterada, esto descarta que la infertilidad observada en los grupos experimentales 1, 2 y 3 se deba al traumatismo por inyección.

En el caso de los grupos experimentales 1 y 2, se observó una disminución significativa en la calidad del semen, progresando a una ausencia de líquido seminal en los pacientes del grupo 3, lo que se relacionaría con un severo daño morfohistológico del parénquima testicular. Por lo tanto ya en el día 14 posterior a la inoculación de cloruro de calcio al $20 \%$, se puede observar que la calidad del semen disminuye y que la espermatogénesis no presenta un desarrollo adecuado para la formación de espermatozoides.

Nuestro estudio difiere de lo descrito por Navarrete, (1997), quién utilizó clorhexidina al 3\% en dimetil sulfóxido (DMSO) al 50\% inoculada en la cola del epidídimo de caninos, señalando que el volumen espermático no sufrió variación estadísticamente significativa entre los grupos ni dentro de estos, en un tiempo final de 45 días. Fahim et al., (1992) inocularon zinc arginina por vía intraepididimal en caninos, observando variaciones estadísticamente significativas en el caso del volumen espermático.

En el presente estudio se observó una disminución de la concentración espermática, la cual fue estadísticamente significativa en el grupo 2, por lo que se podría indicar que esta concentración posee una relación con el tiempo de inoculación del producto, al comparar todos los grupos en estudio se observó una diferencia de la concentración espermática entre el grupo control y los grupos experimentales, lo que indicaría que a los 7 días post-inoculación existiría una diferencia en esta concentración, la cual es más evidente a los 14 días. Estos resultados concuerdan con lo descrito por Navarrete, (1997) quien observó una disminución estadísticamente significativa de la concentración de espermatozoides al inocular clorhexidina al 3\% en dimetil sulfóxido (DMSO) al $50 \%$ inoculada en la cola del epidídimo de caninos, avanzando de una oligozoospermia a una azoospermia en la cuarta a quinta 31 semana post inoculación, describiendo que concentración espermática también presentaba una disminución de forma gradual y progresiva.
Los resultados de concentración plasmática de testosterona indican que el grupo control no existió diferencia estadísticamente significativa entre los tiempos, lo cual era esperable en el estudio. En los grupos experimentales 1 y 2 tampoco se observó diferencia estadísticamente significativa entre los tiempos, lo cual como lo indican los autores Matamoros et al., (2002) y Cunningham (2003), puede deberse a que la testosterona es una hormona muy variable en el tiempo y presenta una liberación cíclica por episodios durante el día y también en las estaciones del año y posee una vida media variable que va desde los 15 a los 60 días. Tampoco se descarta la probabilidad que algunas variantes como edad, momento del día al tomar la muestra y actividad sexual de los caninos en estudio hayan influido en los resultados finales. En el grupo experimental 3 se observó una diferencia significativa en la concentración de testosterona entre el tiempo inicial y a los 21 días, lo que indicaría que es probable que esta hormona disminuya significativamente cuando el daño morfohistológico del testículo sea severo. En el caso de lo descrito por Jana y Samanta (2007), quienes inocularon cloruro de calcio intratesticularmente a dosis de $5,10,15$ y $20 \mathrm{mg} / \mathrm{kg}$, obtuvieron una disminución en las concentraciones de testosterona en el plasma, indicando diferencias estadísticamente significativas en las dosis más altas (15 y $20 \mathrm{mg} / \mathrm{kg}$ ) luego de 45 días post inoculación.

Los resultados obtenidos en el espermiograma y en la concentración plasmática de testosterona indican que los pacientes castrados intratesticularmente con cloruro de calcio al $20 \%$ presentan bajos índices de fertilidad lo que se va acrecentando en el tiempo, existiendo una infertilidad potencial a los 21 días de la inoculación.

Podemos concluir que el cloruro de calcio al 20\% sería una alternativa más inocua que otros agentes químicos utilizados en otros estudios para lograr la castración química, lo cual indicaría que dicho producto químico podría ser una alternativa en castraciones masivas de machos, provocando la reducción efectiva de la fertilidad, lo que podría ser utilizado en campañas de esterilización para el control de la población canina, ya que es una técnica de fácil realización, sin efectos secundarios y económica. 


\section{Referencias}

1. Corti, L.M. 2003. Evaluación de la capacidad fecundante del semen congelado de perro (Canis familiaris), en ova recuperadas de perras en celo inducido. Memoria de título. Universidad Austral de Chile, Facultad de Ciencias Veterinarias, Instituto de Reproducción Animal. Valdivia, Chile.

2. Cunningham, J.G. 2003. Fisiología Veterinaria. $3^{\text {a }}$ ed. Madrid. 575 p. Elsevier.

3. Dellmann, H. Dieter. 1994. Histología Veterinaria. Zaragoza. 398 p. Acribia.

4. Fahim, M.S., Wana, M.F., Sutcu, Z., y Fahim, R.S. 1992. Sterilization of dogs with intra-epididymal injection of zinc arginine. Contraception. 47: $107-122 \mathrm{p}$.

5. Gartner, L.P., y Hiatt, J.L. 2002. Texto Atlas de Histología. $2^{a}$ ed. U.S.A. 483 p. McGraw - Hill.

6. Geneser, F. 2002. Histología: Sobre Bases Biomoleculares. $3^{\text {a }}$ ed. Buenos Aires. 813 p. Panamericana.

7. Gradil, C.M., Yeager, A. y Concannon, P.M. 2006. Evaluación de los Problemas Reproductivos del Macho Canino. Ithaca NY, USA. $1-7$ p.

8. Jana, K., y Samanta, P.K. 2007. Sterilization of male stray dogs with a single intratesticular injection of calcium chloride: a dose-dependent study. Contraception 75, 390 $-400 \mathrm{p}$.

9. Matamoros, R., Goméz, C., y Andaur, M. 2002. Hormonas de utilidad diagnóstica en Medicina Veterinaria. Valdivia. Arch. Med. Vet. 34 n.2. 14 p.

10. Mora, G., Quezada, M., y Bonati. C. 1991. Estudio clínico y morfopatológico de la castración química en terneros con ácido alfa-hidroxipropiónico. Av. Cienc. Vet. $\underline{6}: 55$ $-61 \mathrm{p}$.

11. Navarrete, Y.M. 1997. Tesis. Castración química en perros machos con Digluconato de Clorhexidina al 3\% en Dimetil Sulfoxido al 50\%, Chillan, Chile. Universidad de Concepción, Facultad de Medicina Veterinaria. $72 \mathrm{p}$

12. Nelson, R.W. y Couto, G.C. 2006. Manual de Medicina Interna de Pequeños Animales. Madrid. 894 p. Elsevier.

13. Santos, E.C. 2007. Tesis. Esterilização de cães com injeção intratesticular de solução à base de zinco. Universidad Federal de Minas Gerais. Escuela de Medicina Veterinaria. 90 p.

14. Seremi de Salud Región del Maule. 2008. Programa sobre tenencia responsable de perros en la comuna de Curico, Talca, Constitución, Linares y Cauquenes. 49 p.

15. Tepsumethanon, V., Wilde, H., Hemachudha, T. 2005. Intratesticular Injection of a Balanced Zinc Solution for Permanent Sterilization of Dogs. Faculty of Medicine, Chulalongkorn University. $\underline{88}$ : 686-9 p. 